\title{
Design, Development and Performance Evaluation of Nano Robot in Traditional Siddha Medicines for Cancer Treatment
}

\author{
Kaviarasu Balakrishnan ${ }^{1, *}$, Sivabalan Arumugam ${ }^{2}$ \\ and Guna Magesan ${ }^{3, *}$ \\ ${ }^{1}$ Department of Electronics and Communication, Sathyabama Institute of Science \\ and Technology, Chennai, India \\ ${ }^{2}$ Rakuten Mobile Inc, Japan \\ ${ }^{3}$ Institute of Advanced Research, Gandhinagar, Gujarat, India \\ E-mail: ellipsekaviarasu@gmail.com; gunamagesan@gmail.com; \\ sivabalan.a@gmail.com \\ ${ }^{*}$ Corresponding Author
}

Received 08 December 2019; Accepted 31 December 2019;

Publication 18 July 2020

\begin{abstract}
This paper reports a design and fabrication of a functional nano robot "Sootha Vennai Parpam- cutâvanai - Parpam - SVP" of 150-200 nm size which behaves more or less like a human physician in nano form inside the body. This nanorobot is a medicine which has artificial intelligence i.e. the distinguishing ability, decision making ability and some basic behavioral properties such as target detection ability, self-propelling capability and handling the infection in the case of breast cancer. This nanorobot can also aid in cancer therapy, site-specific or target drug delivery, and tissue repair.
\end{abstract}

Keywords: Nano robot, medical robotics, artificial intelligence, cancer therapy, infection, breast cancer, Target drug delivery, Sootha Vennai, Siddha medicine.

Journal of ICT, Vol. 8_3, 217-234. River Publishers

doi: 10.13052/jicts2245-800X.833

This is an Open Access publication. (C) 2020 the Author(s). All rights reserved. 


\section{Introduction}

Research on nanorobotics has been getting immense attention in the last few decades [1]. Nanorobotics is an emerging field and it deals with the design, manufacturing, programming, and control of robots of nanoscale [2]. Nanorobotics is a multi-disciplinary subject that involves material science, biomedical engineering, artificial intelligence and clinical medicine dealing with nano scale things at molecular level [3]. It has been suggested for an inter-disciplinary bio-inspired approach to be used to design and fabricate nanorobot [4].

Nanorobotics research has been a challenge to engineering, life sciences, and medicine in developing a fully functional or practical nanorobot for biomedical applications [4]. The main challenge is using traditional engineering approaches to fabricate nanorobots.

Many researchers believe that the medical field would be the primary beneficiary and the applications of practical nanorobotics in the medical field would lead a paradigm shift from treatment to prevention $[1,5]$.

Most of current research discusses nanorobotics as a theoretical or hypothetical nanotechnology engineering concept. So far, there is no universally accepted design for practical nanorobots [3]. However [4, 6] have described some key components of a practical nanorobot.

In recent years, research interest in nano-bio formulations and bio nano robots has been growing. As the name suggests, both nano bio formulation and nano robot have dimensions of nanoscale [5], with sizes comparable to bacteria. Due to their small size, nanorobots can directly interact with cells, and even penetrate into them, providing direct access to the cellular machinery [4].

Some characteristics of a robot include: actuation, sensing, propulsion, intelligence, swarm behaviour, manipulation, signalling, or information processing at the nanoscale [2, 7]. If a nano formulation has few or most of these characteristics, then we may consider that nano formulation to be a nano robot.

In comparison to conventional medicines, nano robots have a number of advantages because of its size. Nanorobots have the capability to sense and act in microscopic environments [8]. In the biomedical field, some current research objectives of nanorobotics include early diagnosis of cancer, neutralisation of viruses, target drug delivery, monitoring and treatment of diabetics, and precise and incision less surgery [4, 9]. Long term clinical studies concerning the fate of many nanomaterials in vivo have not been conducted and the fate of these materials remains unknown. 
Siddha - cittã medicine [10] is one of the ancient, traditional systems of medicine originated in India. It is very predominant in the southern part of India, especially in the state of Tamil Nadu, although it is practised in some places across Asia, including Sri Lanka, Malaysia and Singapore. Siddha system has been included in the AYUSH Ministry, Government of India [11]. AYUSH stands for Ayurveda, Yoga \& Naturopathy, Unani, Siddha and Homeopathy - various forms of traditional medicine.

There are more than 120 books written in Tamil - tamil language [12] on Siddha medicine, printed versions of the palm literature, available at the oriental manuscript library, Madras University and published by ThamaraiTãmarai Publishers. The ancient literature on Siddha medicines has identified $n$ number of nano- and organo-metallic compounds[13], such as Vanga Vennai - vañka - vēṇnaiy [14], nano liposomal [15] such as Rasagandhi Mezhugu - ra - kenti - mel̄uku [16] and Gowsigar Kuzhambu - Kaaucikar Kulampu [17]. It is believed that first ever documented nano formulation was given by the great Siddha Agastiyar - akastiyar in his book Agasthiar Paniran Ayiram - agasthiar paṇīirâyiram. The ancient literature has also identified many processes that are used to prepare the nano compounds, with particle size varying from 100-200 $\mathrm{nm}$ [18].

In recent years, a number of research scholars have been working on Siddha based nano medicines. Some of these studies that have characterised nano medicines such as Poorna Chandrothayam - pūraṇa - cantirōtayam [19], Sangu Chunnam - cañku - cuṇnam [20], and Singi Senthuram ciñki-centūram [21].

In the Siddha system of medicine, all nano-bio formulations or medicines are given to the patients based on their body mass ratio, depending on the formulation. Some formulations are given $1 / 2 \mathrm{mg}$ per $1 \mathrm{~kg}$ body weight; some $1 \mathrm{mg}$; and others $2 \mathrm{mg}$ per $1 \mathrm{~kg}$ body weight. For example, a patient with $65 \mathrm{kgs}$ will be given $65 \mathrm{mg} /$ dose/delivery for nano metallic/mineral sulphides (Senthuram-centūram), and about $120 \mathrm{mg} /$ dose/delivery for nano metallic/mineral oxides (Parpam-parpam). However, depending upon the degree of intensity of the disease decides the dose of the drug, supporting drug and the carrier. The typical carriers are ghee, honey and castor oil.

There are more than 330 mercury based formulations/organo metallic formulation in 120 available siddha literature. Among the library have identified few nano materials having robotic behaviours. But we decided to experiment with Sootha Vennai Parpam [22-27], classified as Siddha Sastric drug by AYUSH Ministry, Govt of India, because this single compound has the ability to treat more than 43 prime diseases of different nature. 
In this paper, we focus on the design and development of biologically inspired nano robot $S V P$ - A mercury based nano bio material. Some typical application scenarios such as targeted drug delivery (ability to detect and distinguish the target; self-propelling capability) was developed and studied, which will provide a stepping stone for more research into nanomedical applications.

\section{Materials and Methods}

There are a number of techniques/methodologies available for synthesizing nanomaterials in Siddha system of medicine. They include: 1. combination of grinding and sublimation; 2. combination of grinding and heat treatment; and 3 . grinding and treatment with acids.

The instruments/equipment used to produce the nano robot were "kalvam" (Figure 1(a)) made out of hard granite. The standards dimension for the kalvam mentioned in siddha literature recommends $390 \mathrm{~mm} \times 300 \mathrm{~mm} \times$ $150 \mathrm{~mm}$ [28]. The specially made earthen clay pots (Figures 1(b) and 2) with highest possible density and low porosity. The standard clay port dimension $270 \mathrm{~mm} \times 285 \mathrm{~mm}$.

In this study, the traditional method used to synthesize the nanorobot $S V P$ was by using the combination of grinding and sublimation technique (Figure 1).

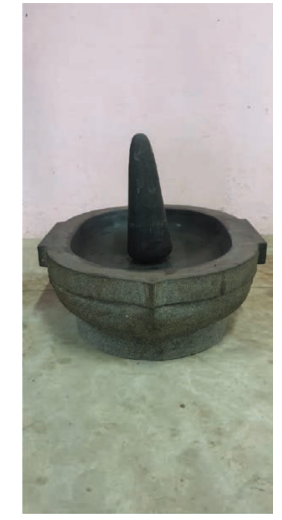

(a)

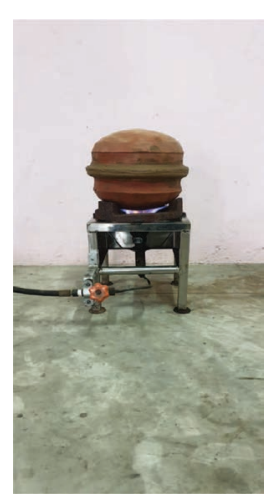

(b)

Figure 1 (a) Combination of grinding and (b) sublimation technique involved in the synthesis of bio nano material, SVP. 


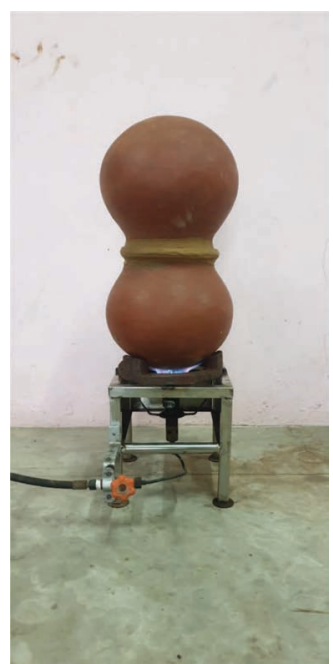

Figure 2 Thiruneelaganda Valai.

The fabrication of the nanorobot $S V P$ was carried out in two phases. In the first, preparatory phase, two ingredients were used to extract mercury. Good quality cinnabar (mercury sulphide) - $700 \mathrm{~g}$ was procured from a mineral supplier. It was made into fine powder using the traditional grinding stone. The second ingredient is Plumbago Zeylanica [28] which was collected from mountain areas. Entire plant was cleaned, dried and powdered (1400 g).

The mercury for the $S V P$ was extracted from cinnabar by distillation process using a sublimation chamber Thiruneelakanda Valai - tirunīlakantavālai (Figure 2) as suggested by Soothamuni-cētamuni. As shown in Figure 2, two earthen vessels made out of clay were placed one over the other. In the lower vessel, 1400 grams of dried Plumbago Zeylanica [28] was taken along with $700 \mathrm{~g}$ of cinnabar. At first, nearly half of the herbal powder was spread evenly at the bottom of the earthen vessel; then the powdered cinnabar was then spread over the herbal powder and finally, the remaining herbal powder was spread on top.

Note that the inner side of the upper earthen vessel has been coated with the herbal extract of the Piper betle [28] and dried in sunlight 3 times to increase the surface tension of the top surface which can collect and hold the sublimated mercury. Mouths of both earthen vessels were joined together after smoothening to avoid any air leakage. The vessels were fitted using clay and cotton. To get the best possible sealing 7 layers of sealing has been done. 
The sublimation chamber has been kept on lotus flame (a flame resembling lotus in appearance generated using three (3 nos) neem wood of $30 \mathrm{~cm}$ in length and 1 inch thickness) for 24 hours. During the burning process, thick wet cotton is placed on the top surface of the upper earthen vessel and wetness is maintained for 24 hours using water. This ensures the upper earthen vessel temperature is less than the lower one and thus the condensation. The chamber has been cooled to room temperature and carefully opened. The low-density sulphur vapor escapes through the micropore of the upper clay pot and the high-density mercury bonded with carbon are deposited as a layer in the inner surface of the upper earthen vessel.

The sublimated material is carefully collected using the brush made out of pig's hair. By applying pressure, followed by water wash, the bonded carbon is removed from the compound and the mercury is separated.

In the second phase, the fabrication of the nanorobot was carried out.

Ingredients used were:

1. Distilled Mercury: $350 \mathrm{~g}$

2. Leaves of Azadirachta indica: Q s

Step 1: Arrange an incinerator towards the northeastern direction. Generally, the physical location of Tamil Nadu state in India which gets cold breeze from north east direction hence the location has been chosen in such a way to get the natural ventilation.

Step 2: Keep the purified mercury in a large earthen vessel.

Step 3: Spread the purified leaves of Azadirachta indica in that earthen vessel above the purified mercury

Step 4: Fill with water inside till the level of the widened mouth of that earthen vessel.

Step 5: Place the earthen vessel inside the incinerator; maintain the flame constantly for 24 hours.

Step 6: Continue the burning process until the water is fully evaporated and collect the mercury from the bottom of the vessel.

Step 7: Again, repeat the process in a new earthen vessel in previous method.

Step 8: Repeat the same process for 41 days using those two earthen vessels.

Step 9: After 41 days, the reddish orange colored drug will be obtained. Keep the drug properly in a hermetically sealed container. 


\section{Measurement}

The SEM: FE with EDX from Carl Zeiss AG, SUPRA 40 with 1.3nm@15kV resolution, 12-900,000 $\times$ magnification, thermal field emission type emitter with acceleration voltage $0.1-30 \mathrm{kV}$, propel current $4 \mathrm{pA}-10 \mathrm{nA}$, high efficiency In-lens sector \& Everhart-Thornley secondary electron detector used for the analysis and characterisation of the Nano robot. The FESEM micrograph in Figures 3.1-3.3 shows having an average diameter of around $30 \mathrm{~nm}$. FESEM micro structural observation from various regions shows size of the particles are in the range of $10-50 \mathrm{~nm}$.

\section{Vehicle}

SVP $240 \mathrm{mg}$ mixed with cow ghee and delivered to the empty stomach after sunrise when the breath runs through the right nostril.

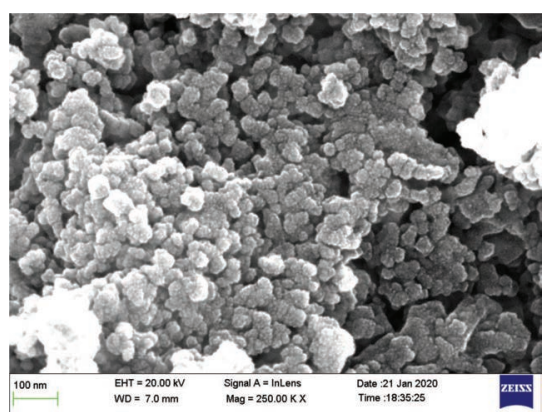

Figure 3.1 FESEM image of Sootha Vennai.

Quantitative results

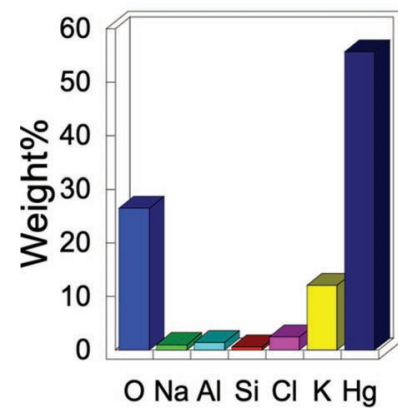

Figure 3.2 Quantitative Analysis of Sootha Vennai. 


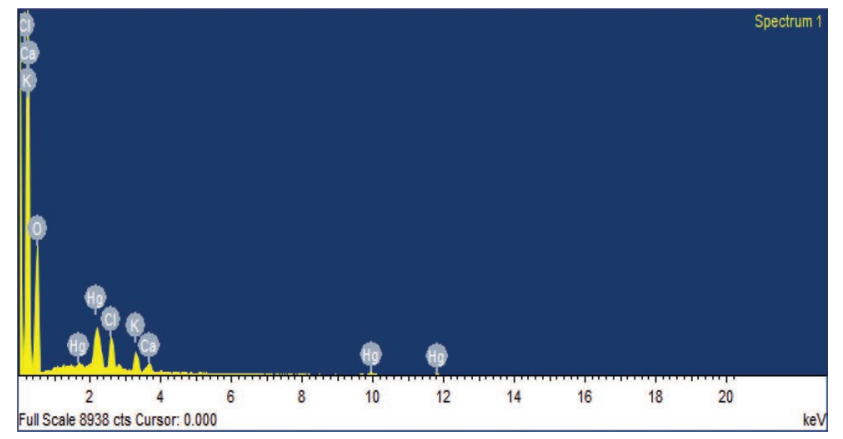

Figure 3.3 Spectrum of Sootha Vennai.

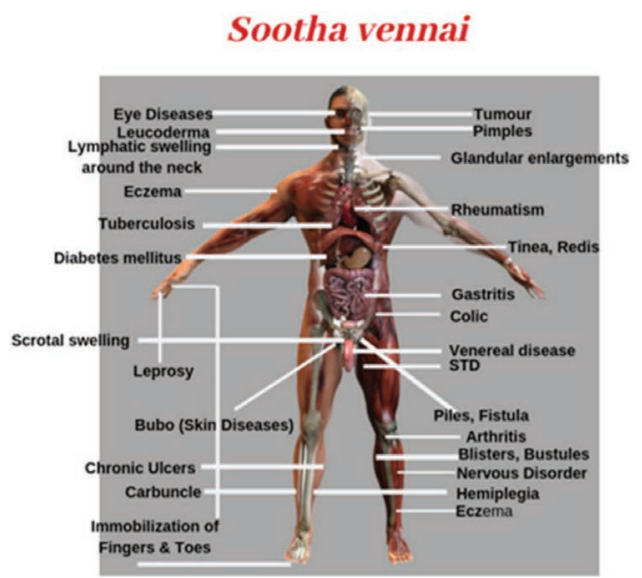

Figure 4 Male Anatomy labeling the activity of the Nano Robot sootha vennai parpam.

Before delivering SVP, $25 \mathrm{ml}$ of thick coconut milk extract has to be taken and after the delivery another $25 \mathrm{ml}$ of coconut milk has to be taken to ensure the SVP particles don't stay in the mouth and reach the stomach.

\section{Indications}

All types of gastritis (peptic ulcer) Figure 4, leprosy, 18 types of colic, 21 types of group of STD diseases, Diseases due to venereal disease, glandular enlargements, bubo (skin diseases), chronic ulcers, sloughy and putrefied ulcers, Carbuncle, cancer-ous tumors, 80 Types of Rheumatism, immobilization of fingers and toes, Hemiplegia, paralysis, 21 Types of nervous disorder, Eye diseases, Cancer in mouth, Kabala head eczema, piles, fistula, 


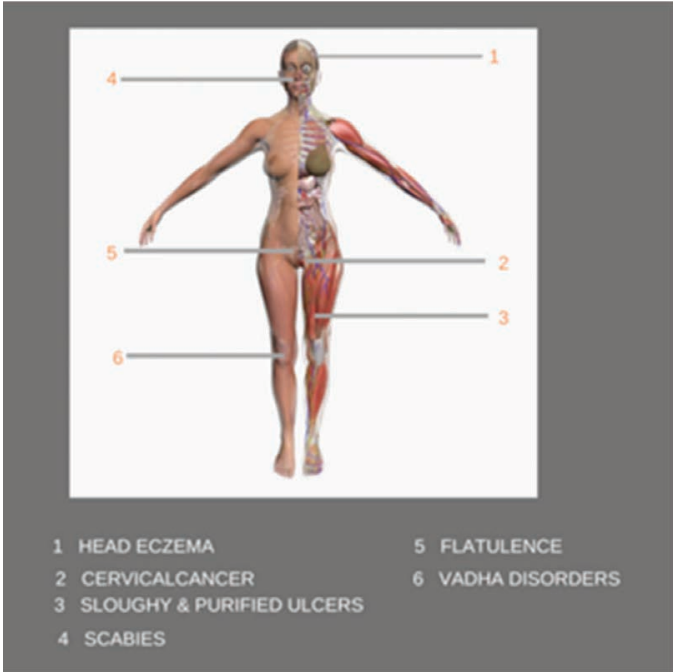

Figure 5 Female Anatomy labeling the activity of the Nano Robot Sootha Vennai parpam.

Eczema, (Erysipelas), itching (skin diseases), scabies, venereal diseases, scrotal swelling, cervical cancer Figure 4, pimples, tinea pedis, blister, pustules, phrynoderma, arthritis, tumour, lymphatic swelling around the neck, Flatulence, Neurology disorder Tuberculosis, diabetes mellitus, polyuria, polydipsia, skin diseases, leukoderma. In addition to that it strengthens the body, boosts the energy levels, act as a rejuvenator.

\section{Case Study}

\section{Case Presentation}

A. A 54 year old woman had pain in the left breast for a month.

B. The patient was admitted to Cancer Institute, Chennai and diagnosed on 10th July 2014. As per Radiation Oncologist the patient was in a critical stage and they are not sure about her treatment results.

C. The patient was taken back to Salem and continued some unknown traditional Siddha medicines from Shimoga for 1 year, private hospital, Salem.

D. On 17.04,2015 she was admitted to private Cancer Institute for treatment, she has undergone Chemotherapy 2 cycles and stopped due to her Psychiatric illness again Chemotherapy for 4 cycles started at 
27.06.2016 to 21.12.2016. Following the treatment patient administered with T.Emeset $4 \mathrm{mg}$, T.Pan $40 \mathrm{mg}$, T.Sizodon $2 \mathrm{mg}$, T.Tripen $2 \mathrm{mg}$, T.Niotrest $10 \mathrm{mg}$.

E. Left Breast Surgery done on 12.01.2017 at Salem Institute and the patient administered with T.Linica 600mg, T.Thioxip forte, C.Haem up.

F. On 01.03.2017 a patient again was admitted for Radiation in Salem cancer institute. Post Radiation she had a Chemo $2^{\text {nd }}$ line 4 cycles from 06.09.2017 to 18.11.2017 and administered with T.Emeset $4 \mathrm{mg}$, T.Rabicip $20 \mathrm{mg}$, T.Flunil $20 \mathrm{Mg}$, T.Sizodon plus, T.Ativan $2 \mathrm{mg}$. Further she can't tolerate Chemotherapy and stopped medications.

G. Again we Checked in a leading Private Cancer hospital for suggestions on 12.02.2019. As they suggested for PET scan and then consulted to take Proton therapy as a solution though the treatment was not affordable for us hence we stopped.

H. On 22.06.2019, the patient was admitted in another private cancer hospital for $3^{\text {rd }}$ line Chemotherapy 5 cycles. Post 5 cycles of chemo doctor prescribed below medicines to follow. T.Zincovit, T.Emeset $8 \mathrm{mg}$, T.rablet, T.Capecitabine $500 \mathrm{mg}$.

I. On 04.09.2019, the patient was administered with Targeted Therapy in private hospital, Chennai. Post 2 cycles of Therapy (on 09.11.2019) her health went very worse, and the wound in her breast got exploded and started bleeding continuously for 2 days and became big and worms formed inside into it.

\section{Examination \& Diagnosis}

A. On 23.11.2019 patient's daughter visited Manushyaa Blossom Multispecialty hospital, Chennai and briefed about the patients health condition and patients inability to travel.

B. The patient administered with SVP, manufactured by Manushyaa Blossom Pvt Ltd, Chennai, India, A Siddha Pharma Company operating under The Office of State Licencing Authority(IM), Ministry of AYUSH, Govt of India, Mfg.Lic.No.1332/25D, Batch No 2, Mfg Date : Oct, 2019, Exp Date : Sep, 2024.

\section{Nano Robotic Management \& Outcomes}

A. Patient administered with SVP, $120 \mathrm{mg} /$ dose with $10 \mathrm{ml}$ Ghee and $100 \mathrm{ml}$ coconut milk extract once a day for 10 days. 
B. Patient administered with SVP, $120 \mathrm{mg} /$ dose with $10 \mathrm{ml}$ Ghee and $100 \mathrm{ml}$ coconut milk extract twice a day for 10 days.

C. Patient administered with SVP, $120 \mathrm{mg} /$ dose with $10 \mathrm{ml}$ Ghee and $100 \mathrm{ml}$ coconut milk extract twice a day for 28 days.
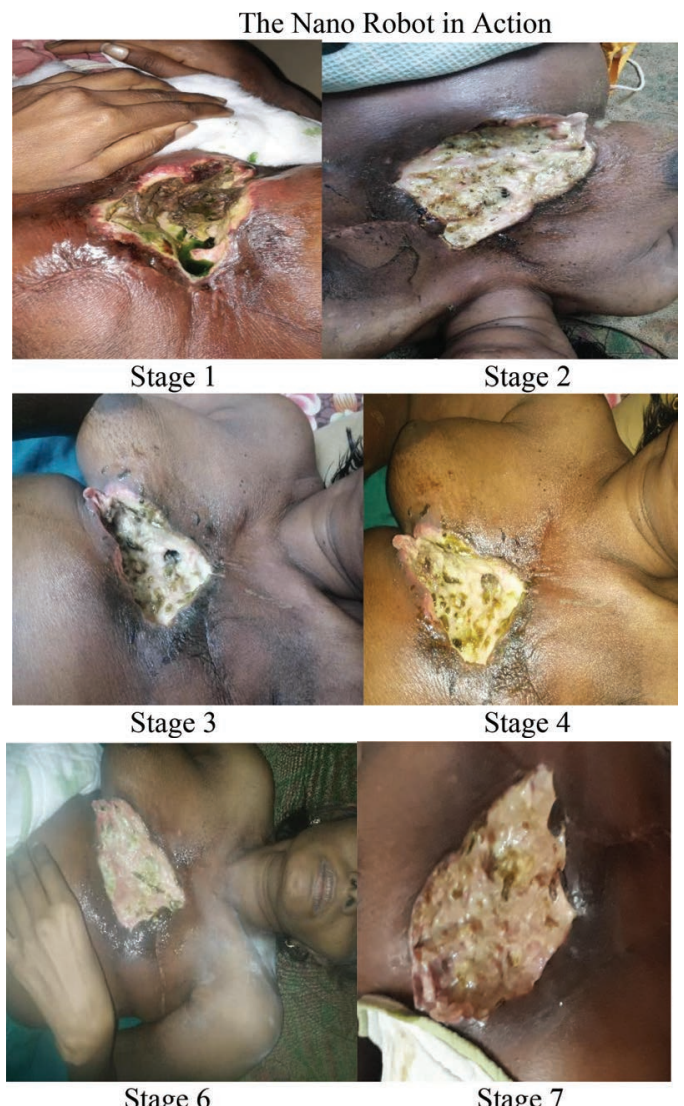

Stage 6

Stage 7

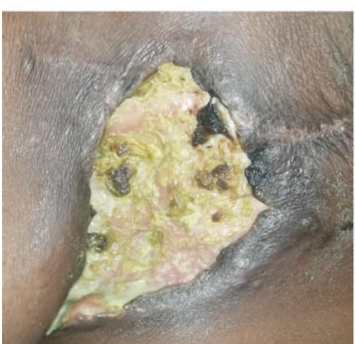

Stage 8

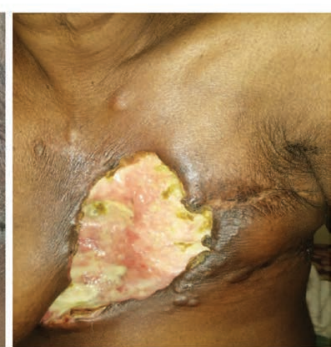

Stage 9 


\section{Results}

The basic objective of the research is to design, produce, certify and test a functional Nano Robot. After successfully producing the Nano Robot having average size of $200 \mathrm{~nm}$ the robotic properties of sootha vennai has been validated using the following procedure.

1. A patient are chosen, one with cancer tumour on the surface of the breast.

2. The Nano robot has been delivered using self-emulsification drug delivery mechanism [30].

3. It demonstrated the ability to detect the tumour on the surface of the breast.

4. The robot was able to propel by itself without any external field support.

5. Temperature rises in the tumour has been detected. Indicating localized working areas of the nano robot. Hence the capabilities of selfpropelling, detecting location, distinguishing cancer cells (indicating intelligence).

6. It has reached the target in the case of cancer tumour and brought down the size of the tumour by one fifth in 20 days' time.

7. The depth of the tumour started reducing and the pus formation has stopped completely.

8. The patient gained enough strength to move around and manage herself.

The particle size of the above formulations play an important role. The smaller the size of the formulation, higher the efficacy. Until early 2011, it was not well known that a section of Siddha formulations were of nano size [31]. When the particle size of the 4 materials Iya Veera Senthuram-ayavira centūram [32], Sandamarutham Senthuram-caṇtamāruta centūram [33], Rasa Sunnam [34] and Rasa Senthuram-raca centūram [35] were measured, atomic force microscopic images gave surprising results used by siddha system for medical application varied between 100-200 nm (Figure 6).

The bright field AFM micrograph in Figure 3 shows the particles having an average diameter of around $200 \mathrm{~nm}$. AFM microstructural observation from various regions shows size of the particles are in the range of 100$250 \mathrm{~nm}$ and most of the particles are measured to be less than $200 \mathrm{~nm}$.

\section{Discussion}

When the scientific community has grand challenges in bioengineered nanorobotics for cancer therapy [36] The people in ancient India had a strong understanding of nanotechnology. 

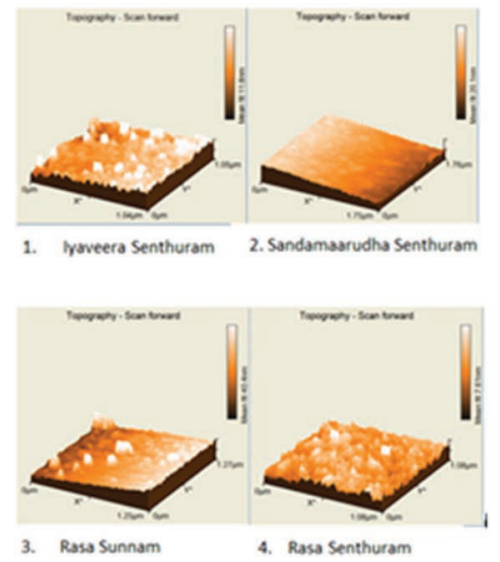

Figure 6 AFM images of Iya Veeram, Sandamarutham, Rasa Sunnam, Rasa Senthuram.

When the current science and technology approach the design and development of nanorobots by designing the power sources, energy harvesting systems, power conditioners, sensors and actuators, embedded control, communication etc., in nanoscale, the scientific community of India (siddha's) approached the same requirements exactly in the opposite direction by designing and synthesizing the molecule with robotic behaviour and artificial intelligence.

The selection of mercury among all the elements present in the periodic table and selection of Leaves of Azadirachta indica out of a few thousand species in the plant kingdom of the southern peninsula of Indian subcontinent is very surprising.

Each and every aspect of the design and fabrication starting from conditioning the clay for fabrication of processing earthen vessel, mercury distillation from cinnabar, choosing materials, synthesize procedure etc., are scientifically well defined.

This study threw new light on the roles of elements like mercury in the human metabolic activity as well as the significance of elements like arsenic and lead in their nano oxide and sulphide forms which in turn indicates their therapeutic significance.

Biological nano robotic systems do exist in nature [5], and our study shows their existence.

The Siddha medical science if researched properly might be able generate the basic guidelines for the usage of nano bio materials to the Drug Agencies across the world about the metabolic activities of metals, minerals 
in their nano forms which leads to the treatment of the most complex health challenges faced by the mankind across the world for the few decades.

In cancer therapy, the basic requirements of a nanorobot are: 1 . The ability to carry a payload, essentially a drug; 2 . Active movement to a specific site in the body; 3. Attachment to the cancerous cells; and 4. Release of the payload locally upon recognition of the binding event. This type of "targeted" therapy had currently eluded investigators, whose present goal is to develop "dumb" nanocarriers, which may attach to cancerous cells by chance [4].

Nanoparticle drug delivery systems have several clinical advantages that make them attractive candidates for the development of a nanorobot core. First, nanoparticle drug delivery systems have clinically been shown to prevent rapid renal clearance and prolong the plasma half-life of complexed or encapsulated drugs [36]. Second, nanoparticles are often more easily endocytosed, which leaves less free drugs available to "normal" cells, reducing harmful side-effects [36]. Third, the high surface to volume ratio of nanoparticles allows for increased drug loading compared to micronsized particles [37]. Finally, nanoparticles have demonstrated the ability to passively penetrate into tumour tissues.

\section{Conclusions}

The SVP nanorobot successfully synthesized, characterized, delivered and demonstrated its capability in handling 4 stage breast cancer tumors. The emulsified SVP delivered by oral means, propelled by itself, reached the target and was repaired. The pus formation completely stopped within 4 weeks and the wound started healing. The SVP should be evaluated further for its ability to carry payload, handle the microbes and other indications.

\section{Acknowledgment}

The Author sincerely expresses his acknowledgment to his master Dr. Krishnamoorthy, RIMP, IMCOPS member for his contribution in the early stages of the development of the nano robot from early 1990's as well as my mother Mrs.Vijayambigai \& Dr. K Foundation for funding the research and development of the Nano Robot. Dr. Krishnamoorthy's love and dedication for the subject has enabled me to proceed with confidence in this nanorobot design. The author would like to thank Mr. Sundra Surian, Malaysia, Directors, Manushyaa Blossom Pvt Ltd, Chennai, India for their support in licensing, manufacturing and the preclinical study. 


\section{References}

[1] Venkatesan, M. and Jolad, B. 2010. Nanorobots in cancer treatment. IEEE.

[2] Ummat, A., Dubey, A., Sharma, G., Mavroidis, C., 2004. "Bio-nanorobotics: state of the art and future challenges," Invited Chapter in The Biomedical Engineering Handbook, 3rd Edition, M. L. Yarmush (ed.), CRC Press.

[3] Elvis S. Liu, On the scalability of agent-based modeling for medical nanorobotics, IEEE Proceedings of the 2015 Winter Simulation Conference, p1427-1435.

[4] Lenaghan, S.C., Wang, Y., Xi, N., Fukuda, T., Tarn, T., Hamel, W.R., and Zhang, M. 2013. Grand challenges in bioengineered nanorobotics for cancer therapy. IEEE Transactions on Biomedical Engineering, 60(3): 667-673.

[5] Requicha, A. A. G., 2003. "Nanorobots, NEMS, and nanoassembly". Proceedings of the IEEE, Special Issue on Nanoelectronics and Nanoprocessing. Vol. 91, No. 11, pp. 1922-1933.

[6] Patel, G.M., Patel, G.C., Patel, R.B., Patel, J.K., and Patel, M. 2006. Nanorobot: A versatile tool in nanomedicine. Journal of Drug Targeting, 14 (2): 63-67.

[7] Mavroidis, C. and Ferreira, A. 2013. "Nanorobotics: Past, Present, and Future'. In Nanorobotics: Current Approaches and Techniques, edited by C. Mavroidis and A. Ferreira. New York: Springer.

[8] Patrick Couvreur and Chirstine Vautheir, "Nanotechnoloogy : Intelligent Design to treat complex diseases", Pharmaceutical Research, Vol. 23, No. 7, July 2006.

[9] Cavalcanti, A., Shirinzadeh, B., and Kretly, L.C. 2008 June. Medical nanorobotics for diabetes control. Nanomedicine: Nanotechnology, Biology, and Medicine, 4 (2): 127-138.

[10] http://siddhacouncil.com/

[11] http://ayush.gov.in/

[12] https://www.britannica.com/topic/Tamil-language

[13] Garima Matela and Robina Aman, 'Tin and OrganoTin (IV) complexes of thymol derivative derived from alanine : synthesis and characteristic spectral studies', RASÃYAN J. Chem, Vol. 4, No. 3 (2011), 594-598.

[14] Siddha vaidiya thirattu, http://www.manushyaablossom.com/siddha-lite rature.php

[15] A. Laouini, C. Jaafar-Maalej, I. Limayem-Blouza, S. Sfar, C. Charcosset, and H. Fessi, "Preparation, Characterization and application 
of Liposomes: State of the Art", Journal of Colloid Sciences and Biotechnology, Vol. 1, 147-168, 2012.

[16] Pullipani, Pulipani-500, Thamari Publishers, Chennai.

[17] Kowsigar, Kowsigar muni nool, Siddha Product Catalogue, IMPCOPS, Chennai.

[18] Agathiyar, Agathiyar 12000, Thamarai Publishers, Chennai.

[19] R. Shailaja, S Sugunthan (year). Concept of nano technology in Siddha medical literature. World Journal of Pharmaceutical Research. Volume 5, Issue 10, 276-284. Review Article ISSN 2277-7105.

[20] K. Arunachalam, K. Rajamaheswari, K. Venkateswaran and K. Suryakumar (year). Anticancer potential of Siddha nano medicine Sangu Chunnam using Mtt assay against Hepg2 (liver cancer cell line). World Journal of Pharmacy and Pharmaceutical Sciences.Volume 8, Issue 3, 814-817.

[21] K. Rajamaheswari, M. Pitchiah Kumar, V. Banumathi and S. Sudharsan (year). Standardization and analytical evaluation of novel Siddha formulation Singi. chenduram: An approach towards nano drug delivery system. Der Pharmacia Lettre, 2015, 7 (11):295-300.

[22] Bogar, Bogar 700. Thamarai Noolagam, Chennai.

[23] Bogar, Bogar Nigandu 1500, Thamarai Noolagam, Chennai.

[24] Bogar, Bogar 7000,Thamarai Noolagam, Chennai.

[25] Bogar, Bogar Janana Sagaram, Thamarai Noolagam, Chennai.

[26] Soothamuni, Soothamuni Soothiram, Thamarai Noolagam, Chennai.

[27] Pullasthiyar, Pullasthiyar Vadha Soothiram, Thamarai Noolagam, Chennai.

[28] Medicinal Plant Of India, Vol-II, Tamil Nadu, S.N.YoganaRasimhan, Year 2000.

[29] Agathiyar, Agathiyar 12000, Vol I, Thamarai Publish-ers, Chennai

[30] Ying Wang, Ying Li, Jian Wu1, Qi Shen (2012). Cha-racterisation and evaluation of self-microemulsifying drug delivery system of Brucea javanica oil. Micro \& Nano Letters, 2012, Vol. 7, Iss. 3, pp. 256-261.

[31] R. Manickavasagam, C. Senthamil Rajam (year). Siddha system and nano technology - a review. Inter-national Journal of Research in Pharmaceutical and Nano Sciences. 5(4), 2016, 235-238.

[32] Agastiyar Kanma Kandam, Kowmathi Nool, Thamarai Publishers, Chennai.

[33] Agastiyar Vaithiya Kaviyam-1500, Thamarai Publishers, Chennai.

[34] Agastiyar Vagara Choothiram-200, Surukam- 100, Thamarai Publishers, Chennai. 
[35] Karuvoorar Vatha Kaviyam 700, Thamarai Publishers, Chennai.

[36] Scott C. Lenaghan, Yongzhong Wang, Ning Xi, Toshio Fukuda, Tzyhjong Tarn, William R. Hamel, and Mingjun Zhang (year). Grand challenges in bioengi-neered nanorobotics for cancer therapy. IEEE Trans-actions On Biomedical Engineering, VOL. 60, NO. 3, MARCH 2013.

[37] Davis, M.E., Chen, Z.G. and Shin, G.M. 2008. Nano-particle therapeutics: An emerging treatment modality for cancer. Nature Rev Drug Discovery, vol 7. pp. 771-782.

\section{Biographies}

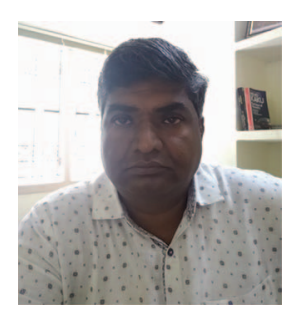

Kaviarasu Balakrishnan received M.Tech in Electronics and Communication Engineering from Pondicherry University, Pondicherry, India in 2000. $\mathrm{He}$ has 24 years of experience in Research and Academic. Presently he works as a Head of Research in Manushyaa Blossom Pvt Ltd, Chennai based Siddha Nano Bio Pharma company. He founded Dr Krishanmoorthy Foundation for Advanced Scientific Research, Vellore, a center for Nano Science in the year 2011. He has designed and synthesized more than 20 nano bio materials and discovered more than 300 processes of nano medicine synthesis using siddha medicine principles. He belonged to the tradition of Indian siddha system of medicine practitioners for more than 700 years. His is specialized in child immunisation using nano formulations. His research interest includes nano robotics, nano drug design, synthesis, delivery and nuclear physics. 


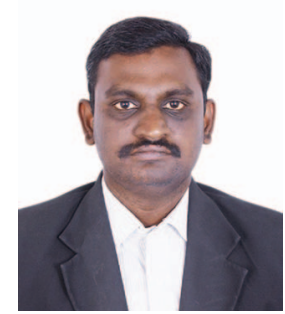

Sivabalan Arumugam received Ph.D in Electrical Engineering from Indian Institute of Technology Kanpur, India in 2008 and M.Tech degree from Pondicherry University, India, in 2000. He has 14 years of experience in Academic teaching and Research. Presently he works as Assistant General Manager for Research at NEC Mobile Network Excellence Center (NMEC), NEC Technologies India Pvt Ltd, Chennai. Prior joining NECI he was associated with ABB Global Services and Industries Limited, Bangalore as Associate Scientist. He has published more than 25 papers in various International Journals and Conferences and also participated in many National and International Conferences. In his current role, he is representing NEC for Global ICT Standards forum of India (GISFI). His research interest includes Next Generation Wireless Networks.

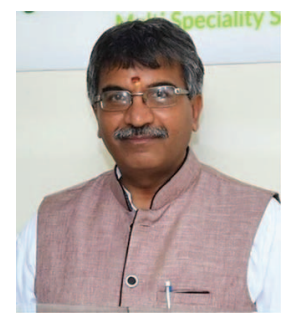

Guna Magesan Director, International Operations, Manushyaa Blossom Pvt Ltd Till recently, Prof Guna Magesan was the Vice Chancellor of Institute of Advanced Research in Gandhinagar, Gujarat. He was the CEO of World Hindu Economic Forum (WHEF) in 2014 and now the Governing Council member of WHEF. Previously, he was the Professor and Head of Environmental Science Dept in Fiji National University. Prior to that, he was a Senior Scientist with New Zealand Govt research organization. He has been actively involved in social and community projects and he was made the Justice of the Peace in New Zealand. 